\title{
APLICAÇÃO DE ZEÓlITAS NaZSM-5 e HZSM-5 COM BAIXA RAZÃO Si/AI NA DESIDRATAÇÃO DO GLICEROL
}

\author{
C. P. ROLDÃO, T. M. NEVES, J. O. FERNANDES, C. A. DA ROSA, V. B. MORTOLA
}

Universidade Federal de Rio Grande, Escola de Química e Alimentos

E-mail para contato: thais.tneves@gmail.com

\begin{abstract}
RESUMO - O glicerol é o principal subproduto da produção de biodiesel. A partir da desidratação catalítica do glicerol é possível a obtenção de acroleína, a qual consiste em um composto utilizado na síntese de produtos com alto valor agregado como o ácido acrílico. Neste trabalho, a zeólita do tipo ZSM-5 com razão $\mathrm{Si} / \mathrm{Al}$ de 25 foi sintetizada na forma sódica (NaZSM-5). O catalisador foi caracterizado por meio das técnicas de DRX, MEV e FTIR. Essa zeólita foi convertida para a forma ácida (HZSM-5) por meio de troca iônica com $\mathrm{NH}_{4} \mathrm{Cl}$. As zeólitas nas formas sódica e ácida foram aplicadas na reação de desidratação do glicerol. Foi verificado que ambos materiais apresentaram atividade nessa reação. No entanto, o catalisador HZSM-5 apresentou maiores valores de conversão de glicerol e seletividade para acroleína. Esse comportamento pode ser atribuído à presença de sítios ácidos de Brønsted, conforme indicado pelos espectros de FTIR desse material. O método de síntese e o procedimento de troca iônica empregados se mostraram favoráveis a obtenção de zeólita na forma ácida com baixa razão $\mathrm{Si} / \mathrm{Al}$ para a produção de acroleína a partir da reação de desidratação do glicerol.
\end{abstract}

\section{INTRODUÇÃO}

Nos dias atuais o uso de fontes alternativas de energia é de extrema importância, devido à poluição ambiental causada pelo uso de combustíveis convencionais e ao aumento do preço dos mesmos (Sannita et al., 2012). Neste contexto, o uso de biodiesel se mostra viável, pois esse biocombustível é biodegradável e produzido a partir de matérias primas de fácil obtenção, como diferentes tipos de lipídios vegetais e gorduras animais (Ong et al., 2011).

No processo químico realizado para a obtenção do biodiesel são gerados subprodutos, sendo o principal desses o glicerol. A utilização desse subproduto para a síntese de produtos de maior valor agregado tem recebido atenção, devido ao glicerol ser bio-sustentável, não tóxico e biodegradável. A obtenção de acroleína a partir do glicerol se mostra mais sustentável em relação ao processo convencional de produção desse composto, o qual consiste na oxidação parcial do propileno. A acroleína é utilizada na produção de polímeros, detergentes e de ácido acrílico (Kiakalaieh et al., 2014).

A desidratação do glicerol a acroleína é realizada na presença de catalisadores ácidos. Nessa reação, a formação de acroleína ocorre quando há interação de um grupo hidroxila secundário da molécula de glicerol com sítios ácidos de Brønsted presentes no catalisador. Vários catalisadores podem ser utilizados, como sulfatos metálicos, fosfatos metálicos, zeólitas, entre outros (Decolatti et al., 2015). As zeólitas são materiais cristalinos e porosos que são formadas por estruturas tetraédricas de $\mathrm{SiO}_{4}$ e $\mathrm{AlO}_{4}$, as quais encontram-se unidas por átomos de oxigênio (Kiakalaieh et al., 2014). Em zeólitas, os sítios ácidos de Brønsted são 
identificados como grupos hidroxila ligados em ponte a átomos de silício e alumínio (Armaroli et al., 2006) e uma elevada densidade desse tipo de sítio é observada em zeólitas com baixas razões $\mathrm{Si} / \mathrm{Al}$ (Wu et al., 2013).

Os objetivos do presente trabalho são a síntese de zeólitas NaZSM-5 e HZSM-5 com razão $\mathrm{Si} / \mathrm{Al}$ de 25 e a aplicação desses catalisadores na reação de desidratação do glicerol visando a produção de acroleína.

\section{MATERIAIS E MÉTODOS}

A síntese da zeólita ZSM-5 na forma sódica com razão Si/Al de 25 (NaZSM-5) foi realizada de acordo com a metodologia de Mortola et al. (2010) e a caracterização dos materiais foi realizada por meio das técnicas de difração de raios-X (DRX), microscopia eletrônica de varredura (MEV) e espectroscopia de infravermelho com transformada de Fourier (FTIR). A obtenção da zeólita na forma ácida (HZSM-5) foi realizada por meio do procedimento de troca iônica de NaZSM-5 com $\mathrm{NH}_{4} \mathrm{Cl}$ (Silva, 2012). Esses materiais foram caracterizados por FTIR.

As zeólitas NaZSM-5 e HZSM-5 foram aplicadas na reação de desidratação do glicerol, conduzida em fase gasosa. No sistema reacional utilizado, uma solução aquosa de glicerol de concentração de $10 \%$ em massa, a vazão de $0,05 \mathrm{~mL} / \mathrm{min}$, foi arrastada por um fluxo de $\mathrm{N}_{2} \mathrm{e}$ alimentada ao reator, o qual se encontrava a $300^{\circ} \mathrm{C}$. O leito catalítico foi preparado com 80 $\mathrm{mg}$ de catalisador. $\mathrm{O}$ efluente do reator foi resfriado a $5{ }^{\circ} \mathrm{C}$ e os produtos líquidos foram analisados por cromatografia gasosa. A conversão de glicerol foi calculada pela Equação 1, onde $\mathrm{m}_{\mathrm{Gin}}$ é a massa de glicerol alimentada ao reator no tempo zero e $\mathrm{m}_{\text {Gout }}$ é a massa de glicerol no efluente do reator em diferentes intervalos de tempo.

$$
\mathrm{X}_{\mathrm{G}}=\frac{\mathrm{m}_{\mathrm{Gin}}-\mathrm{m}_{\text {Gout }}}{\mathrm{m}_{\text {Gin }}}
$$

\section{RESULTADOS E DISCUSSÃO}

\subsection{Caracterização dos materiais}

A Figura 1 apresenta o difratograma da zeólita NaZSM-5. Verifica-se a presença de picos de difração nas posições $2 \theta=7,9^{\circ}, 8,8^{\circ}, 23,1^{\circ}, 23,9^{\circ}$ e $24,4^{\circ}$, os quais são característicos da estrutura MFI da zeólita do tipo ZSM-5 (Shirazi et al., 2008). 
Figura 1 - Difratograma da zeólita NaZSM-5

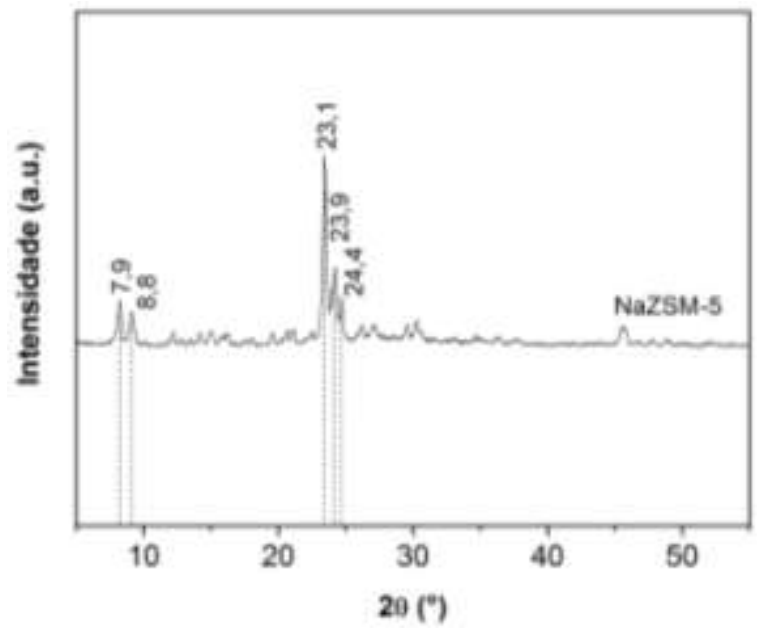

A Figura 2 apresenta a micrografia da zeólita NaZSM-5. Verifica-se que as partículas encontram-se aglomeradas e possuem morfologia esférica. Resultados semelhantes para a zeólita do tipo ZSM-5 são relatados na literatura (Ali et al., 2003).

Figura 2 - Microscopia eletrônica de varredura para a zeólita NaZSM-5

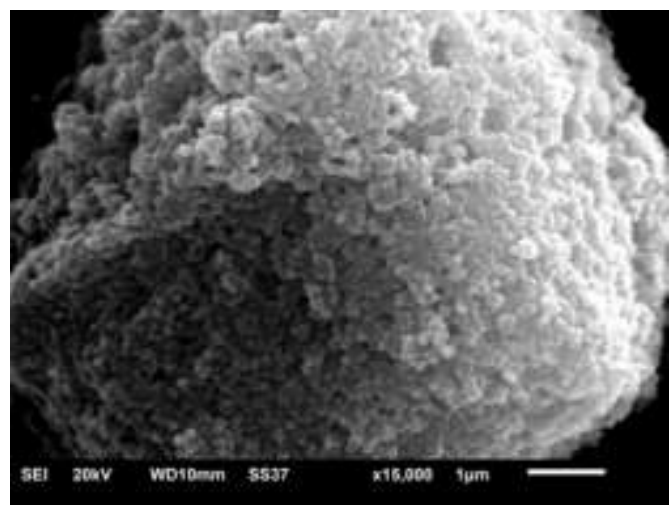

A Figura 3 apresenta o espectro de infravermelho da zeólita NaZSM-5. Observa-se na Figura 3 a presença de uma banda de absorção na posição $450 \mathrm{~cm}^{-1}$, a qual é característica de vibrações de flexão de ligações $\mathrm{T}-\mathrm{O}$, onde T representa um átomo de Si ou Al, que compõem os tetraedros $\mathrm{SiO}_{4}$ e $\mathrm{AlO}_{4}$. A banda em $550 \mathrm{~cm}^{-1}$ é característica das vibrações do duplo anel de cinco membros que fazem parte da estrutura da zeólita. Essas duas bandas de absorção são características da estrutura cristalina da ZSM-5. As bandas de absorção observadas em 790, 1080 e $1220 \mathrm{~cm}^{-1}$ são características de unidades tetraédrica $\mathrm{SiO}_{4}$ que compõem a estrutura da zeólita (Shirazi et al., 2008). 
Figura 3 - Espectro de infravermelho para a zeólita NaZSM-5.

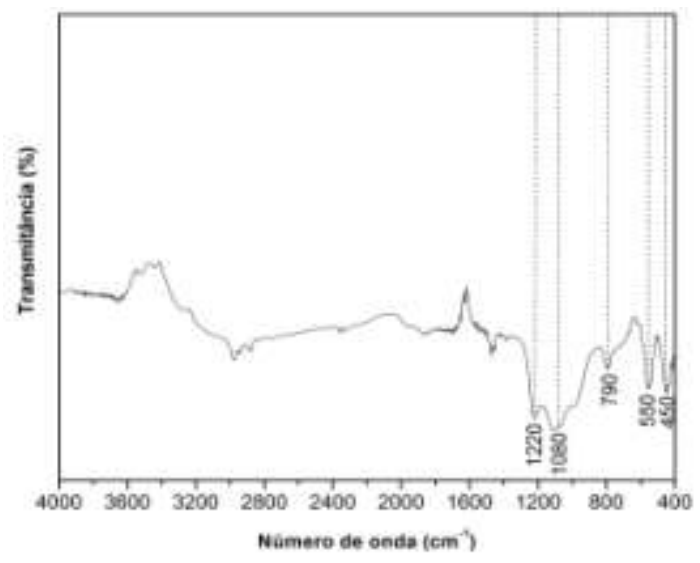

Os espectros de infravermelho de NaZSM-5, da zeólita após a troca iônica e antes do processo de calcinação (ZSM-5 troca $_{\text {) }}$ e de HZSM-5 são apresentados na Figura 4. Verifica-se, no espectro (b) da Figura 4, que duas novas bandas de absorção aparecem após o procedimento de troca iônica. A banda em $1400 \mathrm{~cm}^{-1}$ é característica de ligações $\mathrm{N}-\mathrm{H}$, as quais pertencem a grupos $\mathrm{NH}_{4}{ }^{+}$que se encontram ligados a estrutura da zeólita devido a troca iônica (Ali et al., 2003), enquanto a banda em $3643 \mathrm{~cm}^{-1}$ é característica da presença de sítios ácidos de Brønsted na zeólita (Wu et al., 2013). O espectro (c) da Figura 4 foi obtido após a calcinação da zeólita. Conforme é possível observar, a banda em $1400 \mathrm{~cm}^{-1}$ desaparece após o procedimento de calcinação, devido a decomposição térmica dos íons amônio em amônia gasosa e $\mathrm{H}^{+}$, o qual se encontra ligado a estrutura da zeólita. Já a banda de $3643 \mathrm{~cm}^{-1}$ permanece visível no espectro (c) da Figura 4.

Figura 4 - Espectros de infravermelho para as zeólitas: (a) NaZSM-5, (b) ZSM-5 troca e (c) HZSM-5.

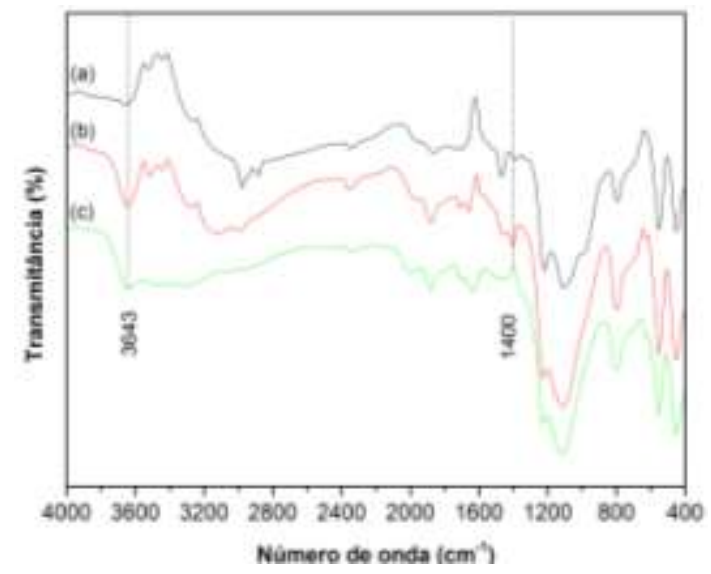

\subsection{Reação de desidratação do glicerol}

A Figura 5 apresenta os resultados de conversão de glicerol com as zeólitas NaZSM-5 e HZSM-5. Verifica-se que os catalisadores sintetizados apresentaram atividade na reação de desidratação do glicerol. Também se observa que a zeólita na forma ácida apresentou desempenho superior ao da zeólita na forma sódica ao longo do tempo. Esse resultado está relacionado a presença elevada densidade de sítios ácidos de Brønsted em HZSM-5. Nas duas 
primeiras horas de reação a conversão de glicerol na HZSM-5 ficou em torno de 99\%. Já na terceira hora, a conversão de glicerol caiu para 83,6\%. Esses valores de conversão são superiores aos relatados em estudos nos quais zeólitas do tipo ZSM-5 na forma ácida foram submetidas a modificações pós-síntese (Wang et al., 2014; Decolatti et al., 2015).

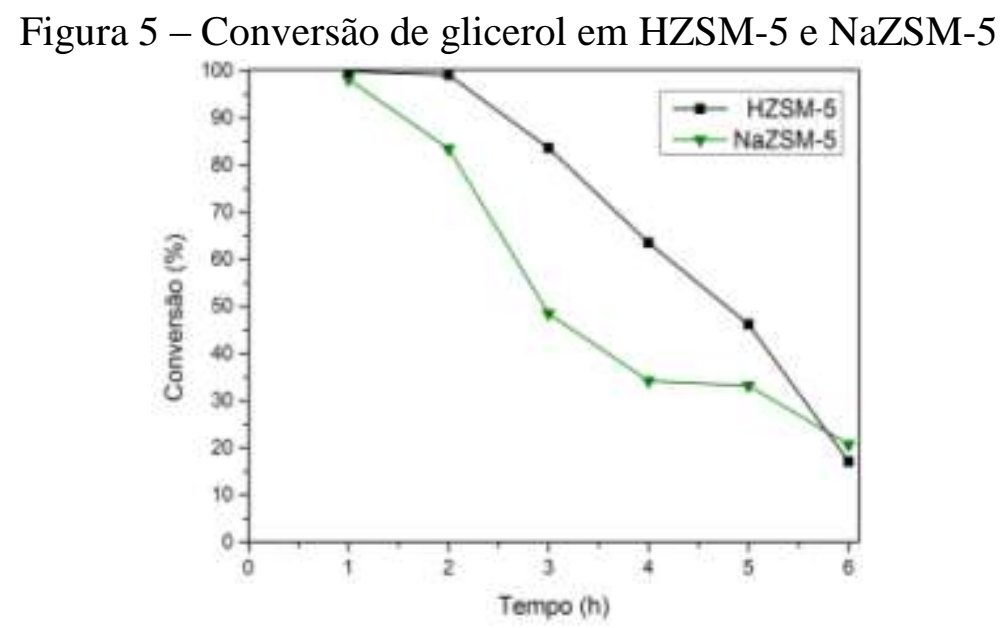

A Figura 6 apresenta o cromatograma obtido por meio da análise do efluente do reator após uma hora de reação com HZSM-5. A acroleína pode ser identificada pelo pico referente ao tempo de retenção de 4,212 minutos, indicando que a reação de desidratação do glicerol utilizando HZSM-5 como catalisador demonstrou-se seletiva para a obtenção desse produto.

Figura 6 - Cromatograma obtido para o efluente do reator

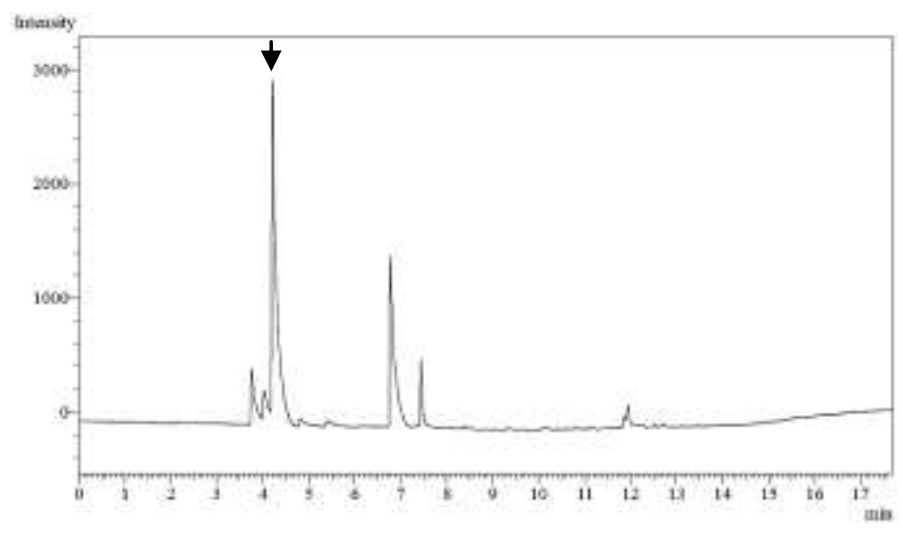

\section{CONCLUSÃO}

Conforme verificado pelas técnicas de caracterização, a síntese da zeólita NaZSM-5 com baixa razão $(\mathrm{Si} / \mathrm{Al}=25)$ e a transformação dessa para a forma HZSM-5 foram conseguidas por meio dos procedimentos utilizados. No espectro de FTIR da HZSM-5, se observou uma banda de absorção referente a presença de sítios ácidos de Brønsted.

Com relação à reação de desidratação do glicerol, as zeólitas NaZSM-5 e HZSM-5 demonstraram elevada atividade e a presença de acroleína foi identificada no efluente do reator. No entanto, maiores conversões de glicerol foram obtidas com a zeólita HZSM-5, evidenciando a importância dos sítios ácidos de Brønsted nessa reação. 
Os resultados de conversão de glicerol obtidos com a HZSM-5 com razão Si/Al de 25 mostraram-se superiores à alguns estudos relatados na literatura. Isso indica que os métodos de síntese e troca iônica empregados no presente trabalho foram efetivos na obtenção de um catalisador promissor para a aplicação na reação de desidratação do glicerol.

\section{REFERÊNCIAS}

ALI, M. A.; BRISDON, B.; THOMAS, W. J. Synthesis, characterization and catalytic activity of ZSM-5 zeolites having variable silicon-to-aluminum ratios. Appl. Catal. A-Gen., v. 252, p. 149-162, 2003.

ARMAROLI, T.; SIMON, L. J.; DIGNE, M.; MONTANARI, T.; BEVILACQUA, M.; VALTCHEV, V.; PATARIN, J.; BUSCA, G. Effects of crystal size and Si/Al ratio on the surface properties of H-ZSM-5 zeolites. Appl. Catal. A-Gen., v. 306, p.78-84, 2006.

DECOLATTI, H. P.; DALlA COSTA, B. O.; QUERINI, C. A. Dehydration of glycerol to acrolein using H-ZSM5 zeolite modified by alkali treatment with $\mathrm{NaOH}$. Micropor. Mesopor. Mat., v. 204, p. 180-189, 2015.

KIAKALAIEH, A. T.; AMIN, N. A. S.; HEZAVEH, H. Glycerol for renewable acrolein production by catalytic dehydration. Renew. Sust. Energ. Rev., v.40, p. 28-59, 2014.

MORTOLA, V. B.; FERREIRA, A. P.; FEDEYKO, J. M.; DOWNING, C.; BUENO, J. M. C.; KUNG, M. C.; KUNG, H. H. Formation of Al-rich nanocrystalline ZSM-5 via chloride-mediated, abrupt, atypical amorphous-to-crystalline transformation. J. Mater. Chem., v. 20, p. 7517-7525, 2010.

ONG, H. C.; MAHLIA, T. M. I.; MASJUKI, R. S.; NORHASYIMA, R. S. Comparison of palm oil, Jatropha curcas and Calophyllum inophyllum for biodiesel: A review. Renew. Sust. Energ. Rev., v. 15, p. 3501-3515, 2011.

SANNITA, E.; ALIAKBARIAN, B.; CASAZZA, A. A.; PEREGO, P.; BUSCA, G. Mediumtemperature conversion of biomass and wastes into liquid products: a review. Renew. Sust. Energ. Rev., v. 16, p. 6455-6475, 2012.

SHIRAZI, L.; JAMSHIDI, E.; GHASEMI, M.R. The effect of Si/Al ratio of ZSM-5 zeolite on its morphology, acidity and crystal size. Cryst. Res. Technol., v. 43, p.1300-1306, 2008.

SILVA, E. D. Formação de mesoporosidade em zeólitas ZSM-5 - Avaliação do método de preparo e do potencial como catalisador ácido através do craqueamento de cicloexano. (Tese de doutorado em Engenharia Química) - Universidade Federal de São Carlos, São Carlos, 2012.

WANG, Z.; WANG, L.; JIANG, Y.; HUANG, J. Cooperativity of Brønsted and Lewis Acid Sites on Zeolite for Glycerol Dehydration. ACS Catal., v. 4, p. 1144-1147, 2014.

WU, G.; WU, W.; WANG, X.; LI, C. Nanosized ZSM-5 zeolites: Seed-induced synthesis and the relation between the physicochemical properties and the catalytic performance in the alkylation of naphthalene. Micropor. Mesopor. Mat., v. 180, p.187-195, 2013. 